\section{Reduction of mixed growth rates in urine by using a "finger tap" method of collection}

Urine bags are still widely used to collect urine from babies for the diagnosis of urinary tract infection, even though bacterial contamination makes this method inappropriate. ${ }^{12}$ It is, however, much less trouble to place a bag over the genitalia than to wait for spontaneous micturition or to subject a child to catheterisation or suprapubic aspiration of the bladder.

The "finger tap" method of stimulating urine flow" provides a simple and reliable alternative way of obtaining "clean" urine for culture. We describe the results of culturing urine specimens obtained from $(a)$ urine bags, $(b)$ "finger tap" clean catch urine from washed genitalia, and $(c)$ finger tap clean catch urine from unwashed genitalia. We also record the time taken to collect urine by the finger tap method.

\section{Subjects, methods, and results}

A total of 154 urine specimens were obtained from 133 children between 1 week and 12 months of age admitted to the National Children's Hospital. Children receiving antibiotics were excluded. Bag specimens were obtained with a Hollister U-bag placed over the genitalia after washing with sterile water and cotton wool. Finger tap specimens were obtained by tapping just above the pubis with two fingers one hour after a feed. Tapping was continued at one tap per second for one minute followed by one minute's rest, and the cycle continued until urine appeared. Samples obtained by tapping were collected directly into a screw cap plastic container. Bag specimens were transferred to a similar container. Specimens were refrigerated immediately. Those obviously contaminated with faeces were discarded.

Cell counts were performed and bacterial counts estimated by a standard loop culture method. ${ }^{4} \mathrm{~A}$ mixed growth of two or more organisms was regarded as indicating contamination. On 48 occasions the time between first tapping the lower abdomen and the appearance of urine was recorded

The table gives the results of the urine cultures. The difference between the washed and unwashed finger tap groups did not reach statistical significance $(0 \cdot 5<\mathrm{p}<0 \cdot 7)$. There was no significant difference in rates of mixed growth between boys ( 11 out of 62$)$ and girls $(16$ out of 71$)(0.5<p<0 \cdot 7)$.

The mean time taken to collect urine by finger tapping was 5.5 minutes $(n=48$, $\mathrm{SD}=6 \cdot 0$ ). The longest time was 20 minutes.

Results of urine cultures in specimens obtained by three methods

\begin{tabular}{lcccc}
\hline \multicolumn{1}{c}{ Collection method } & $\begin{array}{c}\text { No of } \\
\text { urine } \\
\text { samples }\end{array}$ & $\begin{array}{c}\text { Nowith } \\
\text { mixed } \\
\text { growth }\end{array}$ & $\begin{array}{c}\text { \% With } \\
\text { mixed } \\
\text { growth }\end{array}$ & $\begin{array}{c}\chi^{2} \text { test } \\
\text { (comparison with } \\
\text { bag urine) }\end{array}$ \\
\hline Bag with washing & 49 & 25 & $51 \cdot 0$ & \\
Tap with washing & 52 & 4 & $7 \cdot 7$ & $23 \cdot 1(\mathrm{p}<0.001)$ \\
Tap, no washing & 53 & 7 & $13 \cdot 2$ & $16 \cdot 9(\mathrm{p}<0.001)$ \\
\hline
\end{tabular}

\section{Comment}

The $51 \%$ of bag specimens containing mixed growth reaffirms the unsuitability of this method for collecting urine for culture. ${ }^{12}$ The finger tap methods provide an acceptable alternative to bladder aspiration ${ }^{5}$ for routine urine culture.

The items used in the bag collection of urine cost about 30p. Microscopy and culture of a urine specimen costs around $£ 5$ per analysis. Specimens reported as containing a mixed growth are usually repeated. There is therefore a small saving to be made in the cost of urine bags and washing materials if urine is collected by the finger tap method from unwashed genitalia but a much greater saving in laboratory costs.

Most parents can collect a urine specimen by the finger tap method once they have received instruction. Nursing time can be saved if a parent collects urine specimens.

Nurses and parents should not be deterred if the nappy is wet one hour after a feed, as tapping will usually produce a flow of urine. It is also important not to allow the attention to wander, as the flow of urine may easily be missed.

1 Asscher AW. The challenge of urinary tract infections. London: Academic Press, 1980

2 Braude H, Forfar JO, Gould JC, McLeod JW. Cell and bacterial counts in the urine of normal infants and children. Br Med f 1967;iv:697-701.
3 Broomhall $\mathrm{J}$, et al. A reliable, non-invasive technique for obtaining urine samples from babies. $\mathrm{Br}$ Med f 1985;290:30.

$4 \mathrm{McGeachie}$ J, Kennedy AC. Simplified quantitative methods for bacteruria and pyuria. $\mathcal{J}$ Clin Pathol 1963;16:32-8.

5 Arneil AC. Urinary tract infection in children. Br Med J 1985;290:1925-6.

Accepted 20 February 1986

Department of Paediatrics, Trinity College, Dublin, and National Children's Hospital, Dublin 2

M R H TAYLOR, PHD, FRCPI, consultant paediatrician and senior lecturer

National Children's Hospital, Dublin 2

M DILLON, SRN, RSCN, ward sister

Department of Clinical Microbiology, Trinity College, Dublin, and St James's Hospital, Dublin 8

C T KEANE, MB, FRCPATH, associate professor of clinical microbiology

Correspondence to: Dr Taylor, National Children's Hospital.

\section{Sensitivity of neonates to tuberculin after BCG vaccination}

The Department of Health and Social Security recently advised community physicians that BCG vaccination of schoolchildren should stop before 1990 but that vaccination of high risk groups, such as Afro-Asian neonates, should continue.' The World Health Organisation has recommended that vaccination be delayed until three months after birth, because the ability of neonates to produce an adequate immunological response has been questioned. BCG vaccination of neonates is administratively simple and if effective offers protection at an age when the risk of disseminated tuberculosis is highest. Our impression was that sensitivity to tuberculin after BCG vaccination was similar in neonates and children, and we reviewed one year's experience to assess this.

\section{Patients, methods, and results}

In 1984,720 neonates ( $20 \%$ of live births in Derby) were given a BCG vaccination within 10 days of birth by one of three tuberculosis health visitors. Of these neonates, 361 were Asian, 278 had a family history of tuberculosis, and the remainder were vaccinated because they were at high risk or because of parental request. A standard dose of freeze dried vaccine $(0 \cdot 1 \mathrm{ml})$ was administered by Dermo-jet (Mark 9, Schuco International, London) over the left deltoid, and three months later sensitivity to tuberculin was assessed with the Heaf test $(1 \mathrm{~mm}$ setting). In the same year 198 teenagers who yielded negative results on Heaf testing were vaccinated by the same nurses using the same technique.

Heaf tests were performed on $517(72 \%)$ of the infants and $153(77 \%)$ of the teenagers. Those who gave a negative result were retested within one week and revaccinated if the second test also proved negative. The first test yielded negative results for 15 infants and four teenagers. Seven infants had grade 2 results on repeat testing, but all four teenagers remained insensitive to tuberculin. None of the infants yielding negative results were premature or of low birth weight, but six were Asian and two were white, giving a rate of negative results of $2 \cdot 2 \%$ in the Asian and $0.8 \%$ in the white infants. No adverse reactions were seen and no abcesses occurred

\section{Comment}

The effectiveness of BCG vaccination depends on the quality of the vaccine, its transportation, and the technique of its application. The tuberculin state after vaccination is not generally thought to influence the degree of protection offered by BCG; in a group of infants vaccinated in Chicago, however, the only child who did not become sensitive to tuberculin was the only one who died subsequently of disseminated tuberculosis. ${ }^{2}$ The high rate of conversion in those infants may have been related to the technique of multiple puncture vaccination, which appears to require less skill than an intradermal injection using syringe and needle. Grindulis et al found that $50 \%$ of 149 Asian neonates injected intradermally with BCG vaccine gave a negative result on Mantoux testing at 22 months; $25 \%$ had no scar. ${ }^{3}$ In Taiwan only $11 \%$ of vaccinated children who developed tuberculous meningitis before the age of 5 had a BCG scar. ${ }^{4}$ These two reports suggested that either the technique of vaccination was faulty or cell mediated 